\title{
A high-resolution study of $\eta$ Carinae's outer ejecta
}

\author{
Kerstin Weis ${ }^{1,2,3}$, Michael F. Corcoran ${ }^{4,5}$, Kris Davidson ${ }^{2}$, and \\ Roberta M. Humphreys ${ }^{2}$ \\ ${ }^{1}$ Max-Planck-Institut für Radioastronomie, \\ Auf dem Hügel 69, D-53121 Bonn, BRD \\ ${ }^{2}$ Department of Astronomy, University of Minnesota, \\ 116 Church Street SE, Minneapolis, MN 55455, USA \\ ${ }^{3}$ Feodor-Lynen-fellow, Alexander-von-Humboldt foundation \\ ${ }^{4}$ Universities Space Research Association, \\ 7501 Forbes Blvd, Ste 206, Seabrook, MD 20706, USA \\ ${ }^{5}$ Laboratory for High Energy Astrophysics, \\ NASA Goddard Space Flight Center, Greenbelt, MD20771, USA
}

\begin{abstract}
Car is a very luminous and unstable evolved star. Outflowing material ejected during the star's giant eruption in 1843 surrounds it as a nebula, which consists of an inner bipolar region, coined the Homunculus, and the Outer Ejecta. The outer ejecta is very filamentary and shaped irregularly. Kinematic analysis, however, shows a regular bi-directional expansion, despite of the complex morphology. Radial velocities in the outer ejecta reach $2000 \mathrm{~km} \mathrm{~s}^{-1}$ and give rise to X-ray emission, as first detected by ROSAT. We will present a detailed study of the outer ejecta based on HST images, high-resolution echelle spectra for kinematic studies, images from Chandra-ACIS and HST-STIS spectra.
\end{abstract}

\section{Morphology, kinematics and the soft X-ray emission}

The outer part of the nebula around $\eta$ Car contains a countless number of knots, bullets and filaments and manifests the so-called Outer Ejecta (diameter $60^{\prime \prime}$ or $0.67 \mathrm{pc}$ ). A kinematic analysis, using high-resolution echelle spectra, showed that the outer ejecta expand with velocities between $-600 \mathrm{~km} \mathrm{~s}^{-1}$ and $+600 \mathrm{~km} \mathrm{~s}^{-1}$ on average (e.g., Meaburn et al. 1996; Weis 2001a,b). Various filaments nevertheless show much higher radial velocities, e.g., $2000 \mathrm{~km} \mathrm{~s}^{-1}$ (Weis 2001a,b). It is still unclear what triggered the outburst in 1843 and which mechanism not only formed $\eta$ Car's nebula, but also the amazingly high expansion velocities.

With velocities that high, X-ray emission from $\eta$ Car is expected and was indeed detected (e.g., Cheblowski et al. 1984; Corcoran et al. 1997). With ROSAT and Chandra we are now able to separate between a harder, nearly point-like emission from the central source and an extended softer emission from $\eta$ Car's outer ejecta, which is roughly hook-shaped (e.g., Weis et al. 2001). Interestingly, an overlay of the optical image and the X-ray emission shows only a few correlations between the optically emitting gas and the hot X-ray gas (see Figure 1). 


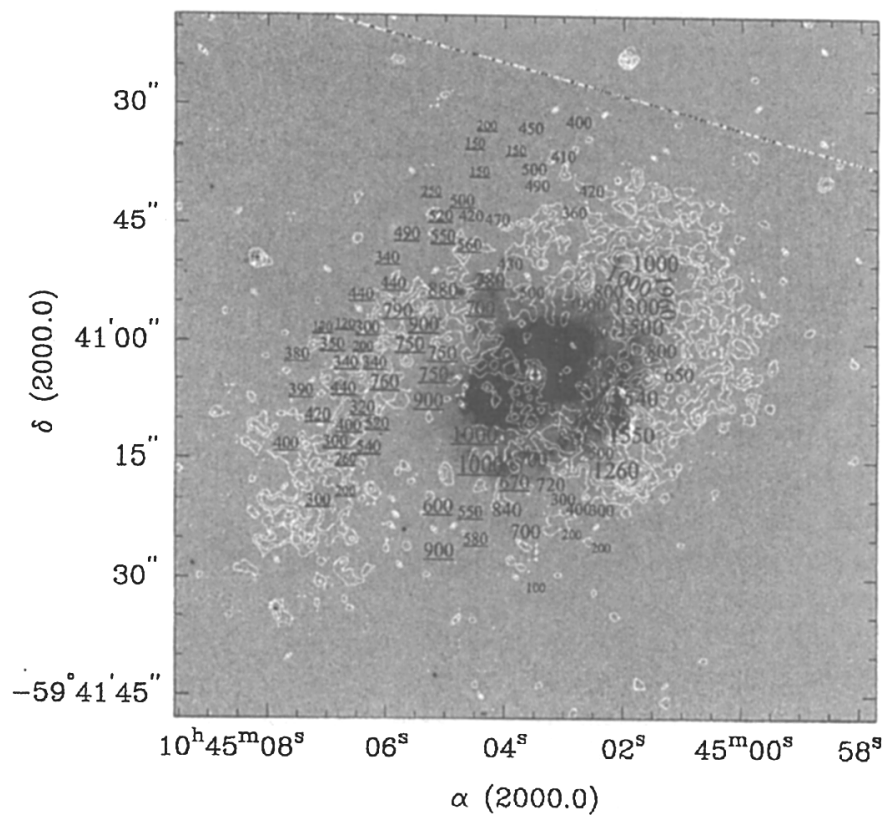

Figure 1. In grayscale the optical (F658N) HST image (WF chip) is shown and in contours the emission in the 0.6 $1.2 \mathrm{keV}$ band as detected with the Chandra-ACIS s3. Numbers indicate the expansion velocities of certain areas, underlined numbers are negative velocities, for better illustration the font size increases for higher velocities.

A much stronger conformance was found in comparison with the radial expansion velocities. The expansion velocities are derived from our optical echelle spectra (FWHM $\simeq 14 \mathrm{~km} \mathrm{~s}^{-1}$ ) and overplotted in Figure 1 with negative velocities underlined. Areas with higher X-ray emission show in general higher expansion velocities. The faster the gas is expanding, the more intense is the X-ray emission. The extended soft X-ray emission from $\eta$ Car and especially the morphology of the X-ray nebula can, therefore, be explained by faster moving filaments which form X-ray emitting shocks. The temperature of the gas (using thermal equilibrium models) is $\sim 0.65 \mathrm{keV}$ (Weis et al. in preparation) indicating postshock velocities of around $750 \mathrm{~km} \mathrm{~s}^{-1}$, in very good agreement with the detected expansion velocities of the bulk of the gas.

\section{References}

Chlebowski, T., Seward, F.D., Swank, J., Szymkowiak, A. 1984, ApJ 281, 665

Corcoran, M.F., Ishibashi, K., Swank, J.H., et al. 1997, Nature 390, 587

Meaburn, J., Boumis, P., Walsh, J.R., et al. 1996, MNRAS 282, 1313

Weis, K. 2001a, in: T.R. Gull, S. Johansson \& K. Davidson (eds.), Eta Carinae and Other Mysterious Stars, ASP-CS 242, 129

Weis, K. 2001b, in: R.E. Schielicke (ed.), Reviews in Mod. Astron. 14, 261

Weis, K., Duschl, W.J., Bomans, D.J. 2001, A\&A 367, 566 